\title{
Automated Analysis of Retinal Images
}

\author{
Piotr Jasiobedzki, Chris J. Taylor \\ Wolfson Image Analysis Unit, Dept. of Medical Biophysics, \\ University of Manchester, Manchester M13 9PT, UK \\ email:pcj@wiau.mb.man.ac.uk
}

\begin{abstract}
We describe a method for segmenting retinal images using positions of blood vessels supplying the retina. The image is tessellated into irregularly shaped primary regions which are bounded by vessels, chains of microaneurysms, edges, etc. Boundaries are classified into groups using a trained set of grey level models. We define a process of merging primary regions into large patches using image properties such as texture and intensity, and semantic interpretations of boundaries and their measured properties. The method which makes extensive use of morphological processing depends on a limited number of parameters which have natural physical interpretations.
\end{abstract}

\section{Introduction and Medical Background}

The optical system of the eye permits direct observation of retinal blood vessels including some capillaries. Diabetes Mellitus, which affects $2 \%$ of the population, is one of the most important diseases which causes abnormalities in the retinal capillaries. This condition, known as Diabetic Retinopathy, is the commonest cause of blindness in developed countries. Changes in the retinal vascular network in diabetes include occlusion of capillaries resulting in non-perfused regions. This process is accompanied by local dilation and deformation of adjacent vessels and formation of microaneurysms, small (25-100 $\mu \mathrm{m}$ in diameter) circular blood filled sacks, which tend to surround areas of non-perfusion.

Fluorescein angiography is an established clinical technique for observing the retinal circulation. Standard images cover a region about $10 \mathrm{~mm}$ in diameter and include the optic disc and/or the fovea. Vessels filled with fluorescent dye are observed as bright ribbons on a non-uniform background. Intensity depends on the quantity of dye, so major vessels can be easily detected, but contrast decreases with decreasing vessel diameter (figure 1). The vessels are roughly cylindrical, and assuming uniform flow of the dye and low attenuation of light by the dye and blood, a profile taken across the vessel can be approximated by a half ellipse. The smallest vessels form a microcapillary net. Separate capillaries can be observed in the region around the fovea where the net is thin and the contrast is good. Further from the fovea where the microcapillary net is much thicker detection of individual capillaries is not feasible.

Analysis of retinal images requires the detection of vessels, microanuerysms and regions of uniform density of capillaries. It is particularly important to identify regions of abnormal perfusion (missing microcapillary network) and relate their positions to the major vessels, optic disc and fovea allowing comparison with the same regions in later images. We have attempted to segment retinal angiograms into 
meaningful regions and to estimate the degree of perfusion (density of microcapillary net) using texture measurements.

\section{Previous Work on Analysing Retinal Images}

Previous work on analysing retinal images has concentrated mainly on detecting vessels and microaneurysms. Tanaka used adaptive thresholding followed by binary thinning to detect major vessels [1]. Similar scheme was been used by Jagoe [2] to detect capillaries in the vicinity of the fovea. Katz [3] segmented and thinned the output from an edge detector to outline vessels. Only major vessels were detected since smaller ones could not be distinguished from other structures. Chaudri [4] described a more robust approach based on detecting local vessel direction by convolving the image with a set of masks. The masks were obtained by rotating a linear feature detector of gaussian cross-section which roughly matched the grey level profile across vessels.

The structure of the vessel tree has been obtained either directly from detected ribbons [3] or from identified branching and crossing points [1]. Vessel segments joining these points were then extracted from the image. Akita [5] described the use of relaxation labelling to provide a connectivity interpretation for the vessel segments and to label vessels as arteries or veins.

Lay detected microaneurysms using a morphological "top hat" transformation [6]. The top hat transformation was performed by subtracting the image opened using linear structuring elements from the original. The resulting image contained objects of circular shape and of diameter less than the structuring element length.

The problem of detecting non-perfused zones has received little attention. Goldberg [7] described results of clustering regions of similar grey level. The image was smoothed and segmented, with a fixed threshold, into perfused and non-perfused regions. We have used [8] texture measures derived by morphological opening and closing of the image to measure degree of perfusion.

\section{Outline of the Method}

The approach we have taken follows the method used in the manual analysis of retinal angiograms by ophthalmologists. Regions which contain a fine and regular structure of capillaries are regarded as well perfused. Areas where this structure is not visible are regarded as non-perfused. Boundaries of such regions are usually aligned with the positions of vessels or are created by joining adjacent groups of microaneurysms and other visible structures in the image.

Automatic segmentation of angiograms requires a representation of data which allows for effective merging and/or splitting of regions and a method for labelling such regions depending on their image properties. Quad-trees and adjacency graphs have been used to represent the image data in split and merge processes [9]. Quadtrees suffer from dependence of the tree structure on initial alignment with the image 
(the structure of the tree may change significantly with a small shift). Also, adjacency between regions is not stored in the quad-tree directly; access to an adjacent pixel may require the whole tree to be traversed. Adjacency graphs, when built from the pixel level, require extremely large amounts of data to describe the links between regions in the graph. A reasonable compromise between the size of the graph and the amount of data necessary to describe it can be achieved, if information on, for example, boundaries is used to create an initial tessellation of the image.

Meyer [10] described a method of tessellating the image into irregularly shaped patches, by detecting watershed lines of the gradient image. The difference in grey levels measured in the centres of regions in the original image was used a merging criterion. Montanvert [11] created the adjacency graph at pixel level and defined a stochastic process for decimation the number of nodes in the graph. For grey level images the decimation process was modified by a local classification using region intensities.

We obtain an initial tessellation of the image into primary regions by detecting linear structures in the image. These boundaries are described by a set of features derived from the original image and labelled using a statistical classifier. Classification uses a set of boundary models specific to this application. The boundaries are modelled as having finite width and are excluded from the definition of primary regions, so the image properties (intensity, texture, etc.) of each region are more uniform.

Since individual capillaries cannot be visualised reliably, we have used texture measurements to characterise the degree of perfusion in each region. The methods we have used are based on morphological sizing, since we are dealing with structures whose dimensions are reasonably well known. Adjacent regions are merged, when they are separated by an appropriate class of boundary and are of similar intensity amd texture. One of the general problems in analysing textured images is reliable identification of the boundaries between regions of different texture. We solve this problem by using non-textural information to define the boundaries.

\section{Detecting and Labelling of Region Boundaries}

A boundary description of a retinal angiogram is built in two stages. First, linear structures corresponding to vessels, lines of adjacent microaneurysms and other structures present in the image are detected, and represented as a network of arcs and nodes. In the next step each of the arcs is processed separately by referring to the original image and classified, accordingly to grey level appearance, into several classes.

The original image (figure 1) is smoothed by morphological opening and resampled giving a low resolution version of the image. The resulting image is morphologically thinned to give one pixel wide lines using a modification of method described by Goetcherian $[12,13]$. Thinning creates plateaus of uniform intensity which 


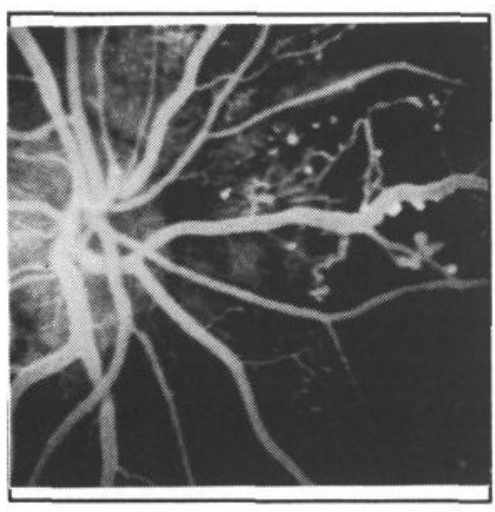

Figure 1. Angiographic image of the retina.

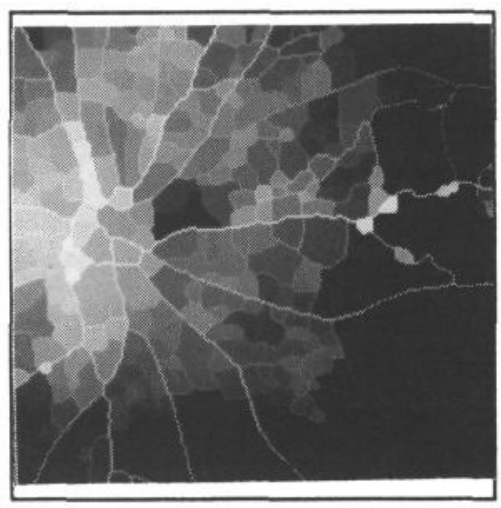

Figure 2. Smoothed, resampled and thinned version of image from figure 1 .

are surrounded by ridges (figure 2 ) which correspond to watershed lines of the image. The thinned image is segmented into 8-connected arcs and 4-connected background in two steps. First the inner points on the plateaus are detected (i.e. points which have all 8-connected neighbours of equal value). Then their 4-connected neighbours are tested to see whether they belong to the plateau (are of equal value) and are labelled accordingly. This method ensures that the arcs are aligned with watershed lines of the image and their connectivity is resolved properly (figure 3 ).

To identify the type of object that each arc represents, it is necessary to refer to the original image and analyse the grey level distribution around the arc. This is performed by generating a sequence of lines perpendicular to the arc and sampling the original image along the lines to obtain grey level profiles. Each line is placed so that it's mid-point lies on the arc. Profiles are convolved with a derivative mask to give gradient profiles. The positions of two extreme values of the gradient, to the left and right of the centre are detected and the fragment of the original profile between the detected points is selected as the most likely location for a vessel. An ellipse is fitted to this section of the profile using a least square error criterion. Figure 4 shows the grey level profile taken across a vessel and the fitted ellipse. For each arc, several profiles are analysed, and parameters such as relative peak height, mean and standard deviation of the width, aspect ratio and the residual error of the fitted ellipses, are derived. These features are used to describe and classify the arcs. We have defined five classes to which each arc can be assigned: large vessels, small vessels, micro-structures, artifacts, borders.

Classes of large and small vessels correspond to vessels of different size: distinction is made primarily on basis of their widths but the aspect ratio of fitted ellipses is also different. Both large and small vessels detected in the network are shown in figure 5. Micro-structures is a fairly wide class, which includes arcs created by groups of 


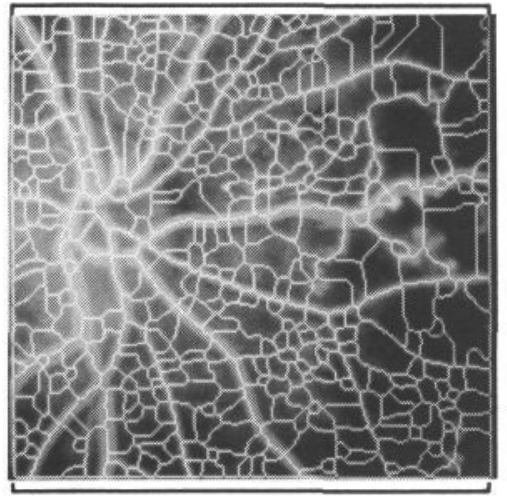

Figure 3. Network of arcs detected in the image from figure 2.

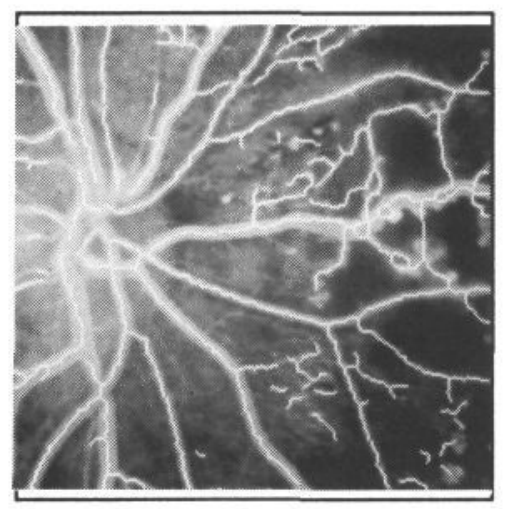

Figure 5. Large and small vessels detected in the image from figure 3.

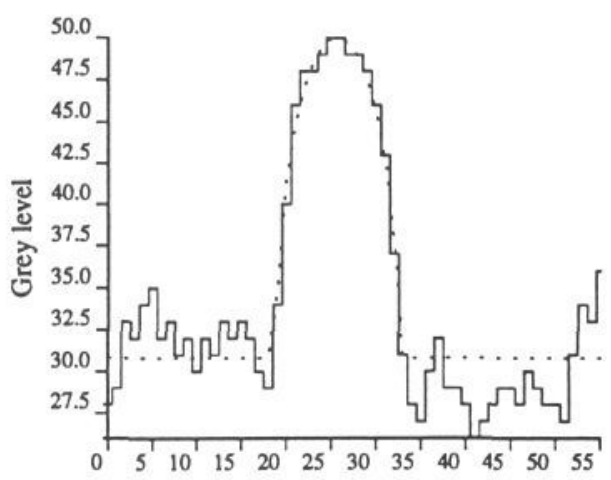

Figure 4. Profile taken across a vessel and it's approximation by an ellipse.

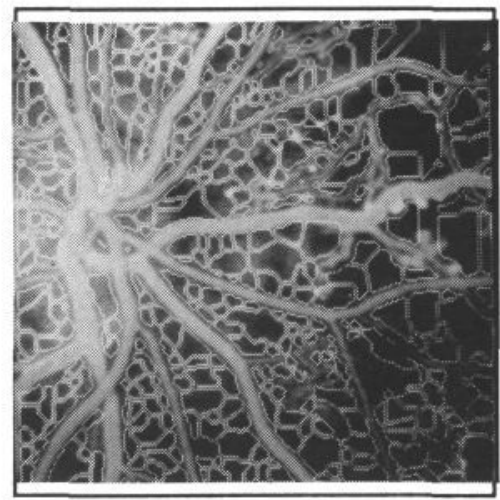

Figure 6. Primary regions detected in the image in figure 2.

microaneurysms, edges, etc. The thinning process inevitably creates watersheds when there are even small valleys on both sides of a plateau. Arcs detected in such situations are classified as artifacts. The borders class corresponds to arcs detected at the edge of the image.

\section{Constructing Primary Regions}

The network of arcs defines regions in a natural way. A region description is obtained by traversing arcs, turning right at each node until the starting arc is reached. This is repeated until all arcs have been used twice (in both directions. The image is then described as a region adjacency graph where arcs correspond to region boundaries (vessels, micro-structures, artifacts, etc), nodes to bifurcation or crossings of vessels 
or other structures. Adjacency of regions and connectivity of arcs is stored explicitly in the graph. The actual extent of each region is defined in such way that it excludes any part of the outlining vessel. This is achieved by reducing regions by the width of adjacent vessels. It is then possible to measure properties of regions directly without any bias caused by the dominating vessel structure. Figure 6 shows the primary regions constructed using the labelled network from figure 5 .

\section{Measuring Texture in Primary Regions}

Morphologically derived features have been used in various ways to characterise texture in binary or grey level images. Werman [14] calculated a feature matrix using linear structuring elements of increasing lengths with different orientation. Serra [13] used random set theory to model texture. Maragos [15] measured entropy of a morphological pattern spectrum. A box measure of a fractal surface, defined by Mandelbrot [16], has also been implemented using morphological operations [17, 18].

The main problem in using morphological operations to describe texture is that it is possible either to use a combination of many measures or to try to model the process which generates the texture, estimate its parameters and limit interest to a class of applications. In our application where we are interested in differentiating between regions which contain dense structure of capillaries and regions where this structure is severely reduced, we have followed the second approach.

Diagrams $a$ to $d$, in figure 7, represent grey level profiles obtained by sampling the image along lines in regions with different degrees of perfusion. Grey-level closing with a structuring element $B_{1}$ fills the valleys in profile $a$ increasing the average

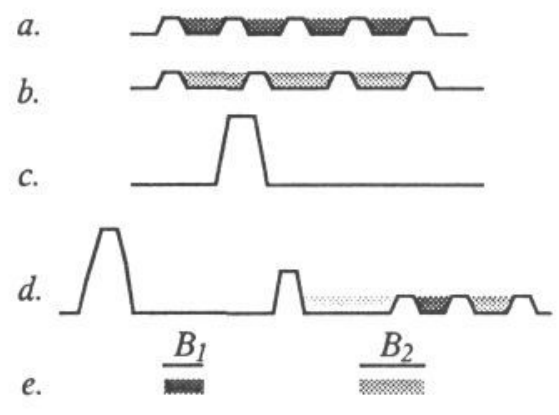

Figure 7. The effect of closing on $1 D$ profile: (a) profile across well perfused region, (b) profile across less perfused region, (c) profile across non-perfused region, (d) profile across a "real" region, (e) two structuring elements of different sizes.

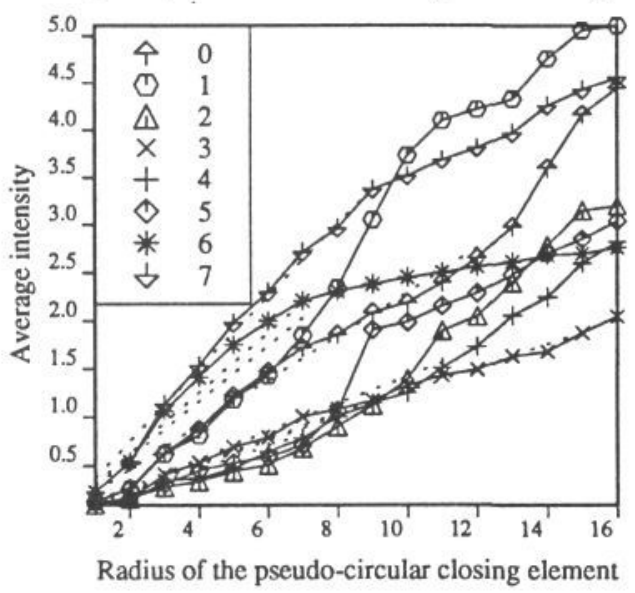

Figure 8. The effect of closing the image on average intensity for selected regions: 2,3 , 4,5 are non-perfused, 0,1,6,7 are perfused. 
grey level but does not change profiles $b$ and $c$. Increasing the structuring element size to $B_{2}$ fills the valleys in profile $b$ but $c$ remains unchanged. For a periodic texture one may expect a significant increase in average grey level intensity for a precisely defined size of the structuring element. In the real case we obtain profiles more similar to $d$. Then, for a region containing a microcapillary network, we obtain responses for a wide range of structuring element sizes. For non-perfused regions we obtain some response due to the presence of noise and other visible structures unrelated to the capillaries.

Figure 8 shows the change in average grey level as a function of the radius of pseudo-circular structuring elements for number of perfused and non-perfused regions. The difference between the closed and the original image is calculated for the whole region and normalised by its size. For non-perfused regions the slope is low, for perfused ones the slope is high. We use the slope of the least square error (LSE) straight line as a feature for classification purposes.

In addition we use the grey-level opening of the image in a similar way in order to measure the size and number of the capillaries in the region. We measure the normalised difference between original and opened images as a function of the structuring element size. The slope of the LSE line fitted to the curves is used as a second feature to describe regions. In practice it is unlikely that these features are truly independent but they encode essentially different properties of the texture. Figure 9 shows one of the features measured in primary regions and linearly mapped into the image.

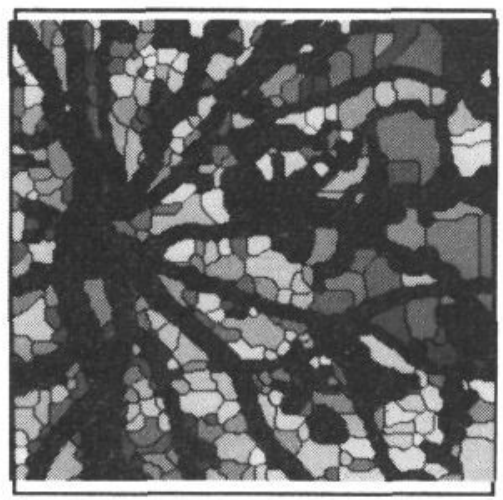

Figure 9. Feature map obtained for primary regions and linearly mapped into the image.

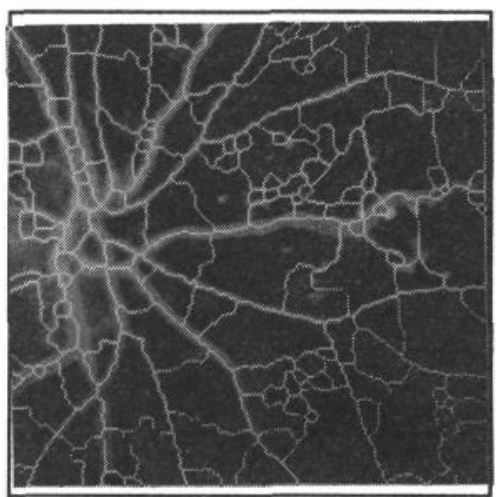

Figure 10. Network after merging regions on basis of their image properties and description of boundaries.

Comparing results of manual grading of regions by an ophthalmologist with such feature maps we have found a good correspondence between large, non-perfused regions in the image [8]. The correspondence decreases for small regions due to the fact that texture is a property which can be measured more reliably in large regions. 


\section{Merging Primary Regions}

Primary regions are merged together on basis of their texture and intensity. It is essential to merge regions in a such way so as not to lose the underlying structure of vessels in the image. This structure provides the reference for defining positions of various features in the image, and allows for final interpretation of the image, and matching of images.

Merging starts by deleting arcs classified as artifacts between regions of similar image properties. Each new region includes all points which belong to the merged regions together with points which belong to the boundary. Properties of the new region are obtained by referring to the original image. Merging of regions separated by a boundary labelled as a micro structure is performed by combining points which belong to both regions but excludes the boundary. Properties of the new region are obtained by weighted averaging of properties of both regions. Deletion of an arc from the network requires examination of nodes where this arc is connected. The node is deleted if there are only two connected neighbours left and the rules of merging arcs are fulfilled. It is obviously possible to merge arcs of the same type but some other combinations are allowed. Connection of arcs requires selecting a proper label and updating the description. Figure 10 shows a reduced network obtained by merging regions of similar intensity and texture, and allowing for deletion of arcs labelled as artifacts only.

\section{Results and Discussion}

There is a single parameter which controls the process of detecting boundary network: the size of structuring element used for opening. This parameter has a natural interpretation for it corresponds to the minimum size of features used for delineating regions and thus indirectly determines the number and size of identified regions. The axial symmetry of vessels ensures that grey level thinning of the image defines the axes of vessels. Classification scheme based on boundary models detects isolated vessels very reliably. There are some problems at places where vessels cross: only one arc is then detected (compare figures 3 and 5). Sometimes the arc is oriented in such direction that the profiles look different and the arc is misclassified. To resolve this properly it is necessary to analyse the whole structure of the vessel tree and use constraints on vessel widths, connectivity at bifurcations and crossings, etc.

The detection of transitional phases between adjacent regions allows image properties such as average intensity and texture to be used without the influence of vessels which dominate the image of the retina. Our approach aligns the regions to the position of vessels and allows us to map detected regions to these elements of the retina which do not change even over a long period of time. The features which are used for grading the images are sensitive enough to detect non-perfused regions. 
Development of the described method of tessellating the image, identifying boundary classes and merging has been motivated by the described application. We can see extension of this research into other medical applications where it possible to define and train models of boundaries, define rules on their connectivity and use this information in merging process.

\section{Acknowledgements}

This research has been supported by a grant from the Kershaw Trust. We are grateful to Prof D. McLeod from Dept. of Ophthalmology. University of Manchester for proving us retinal images and for helpful advice. Dave Bailes and Andrew Hill for programs to detect networks in binary images.

\section{References}

[1] Tanaka M., Tanaka K.: An Automatic Technique for Fundus Photograph Mosaic and Vascular Net Reconstruction. Proc. of MEDINFO 80. Tokyo, pp.116-120.

[2] Jagoe R., Wootton R.: Quantification of Retinal Damage During Cardiopulmonary Bypass: Comparison of Computer and Human Assessment. IEE Proc., vol. 137. pt. I, no. 3 , June, 1990, pp. 170-175.

[3] Katz N., Goldbaum M., Nelson M., Chaudri S.: An Image Processing System for Automatic Retina Diagnosis. Proc. of SPIE vol. 902, 1988. pp.131-137.

[4] Chaudri S., Chatterjee S., Katz N., Nelson M., Goldbaum M.: Detection of Blood Vessels in Retinal Images Using Two-Dimensional Matched Filters. IEEE Trans on Medical Imaging, vol. 8, no. 3, 1989, pp. 263-269.

[5] Akita K., Kuga H.: Pattern Recognition of Blood Vessel Networks in Ocular Fundus Images. Proc. SPIE vol. 375, 1982. pp. 436-441.

[6] Lay J., Badouin B.: Computer Analysis of Angioflourograms. Proc. of VII Int. Conf. on Pattern Recognition, 1984, pp.927-929.

[7] Goldberg E.G., Varma S., Spaeth S.: Quantification of Progressive Diabetic Macular Non-perfusion. Ophthalmic Surgery, vol. 20, no. 1, 1989, pp. 42-45.

[8] Jasiobedzki P., Macleod D., Taylor C.J.: Detection of Non-perfused Zones in Retinal Images. IV IEEE Symposium on Computer Based Medical Systems, Baltimore, May, 1991, pp. 162-169.

[9] Pavlidis T.: Structural Pattern Recognition. Springer Verlag. 1979.

[10] Meyer F., Beuchers S.: Morphological Segmentation. J. of Visual Communication and Image Interpretation, vol. 1, no. 1, Sept., 1990, pp. 21-26.

[11] Montanvert A.. Meer P.. Rosenfeld A.: Hierarchical Image Analysis Using Irregular Tesselations. IEEE Trans. on PAMI, vol. 13, no. 4, April, 1991, pp. 307-316.

[12] Goetcherian V.: From Binary to Grey Tone Image Processing Using Fuzzy Logic Concepts. Pattern Recognition, vol. 12, 1980, pp. 7-15.

[13] Serra J.: Image Analysis and Mathematical Morphology. Academic Press, 1982.

[14] Werman M., Peleg S.: Min-Max Operations in Texture Analysis. IEEE Trans. on PAMI, vol. 7, no. 6, 1985, pp. 730-733.

[15] Maragos P.: Pattern Spectrum and Multiscale Shape Representation. IEEE Trans. on PAMI, vol. 11, no. 7, July, 1989, pp. 701-715.

[16] Mandelbrot B.: The Fractal Geometry of Nature. Freeman, 1982.

[17] Peleg et al.: Multiple Resolution Texture Analysis and Classification. IEEE Trans. on PAMI, vol. 6, no. 4, 1984, pp.518-523.

[18] Rigaut J.P.: Automated Image Segmentation by Mathematical Morphology and Fractal Geometry. J. of Microscopy, vol. 150, 1988, pp. 21-30. 